International Journal of Engineering \& Technology, $7(4.35)(2018)$ 559-563
International Journal of Engineering \& Technology
SPC
Website: www.sciencepubco.com/index.php/IJET
Research paper

\title{
Effect of Multiplication and Absorption Layers Width on Avalanche Multiplication Gain in InGaAs/InP Avalanche Photodiode
}

\author{
Mahdi All Khamis ${ }^{1}$, W. Emilin Rashid ${ }^{2}$, Pin Jern Ker ${ }^{3}$, K. Y. Lau ${ }^{4}$ \\ Institute of Power Engineering, \\ Universiti Tenaga Nasional, \\ Jalan IKRAM-UNITEN, \\ 43000 Kajang, Selangor, Malaysia \\ *Corresponding author E-mail: m-allkhamis@hotmail.com
}

\begin{abstract}
The separate absorption, grading, charge, and multiplication (SAGCM) InGaAs/InP avalanche photodiodes (ADPs) are widely used in long distance, high bit rate optical communication system due to their high performance and response to optical fiber wavelength spectrum. In this work, the effect of multiplication layer width (MLW) and absorption layer width (ALW) on APD performance is studied and investigated. Silvaco TCAD software is used as simulation tools to simulate a precise model of InGaAs/InP APD and analyze its, performance under an illuminated condition. As such, three different ALW with various MLW has been simulated while the structure values and material parameters are kept constant. It was found that in the APD with smaller MLW, the distance between the punch-through voltage and the breakdown voltage can be maximized. Therefore, the operation region of APD will be extended. In addition, the multiplication gain is obtained from the photocurrent and primary current by taking the APD collection efficiency effect under the consideration.
\end{abstract}

Keywords: Multiplication gain, Avalanche photodiodes, Punch-through voltage, Avalanche breakdown.

\section{Introduction}

Avalanche photodiodes (APDs) are attractive devices for several applications such as optical communication, nuclear medicine, and aerospace [1]. Recently, the separate absorption, grading, charged and multiplication (SAGCM) InGaAs/InP APD has been known to be an effective detector for high bit-rate, long distance optical communication application. This is because of the narrow bandgap of InGaAs $\left(E_{g}=0.77 \mathrm{eV}\right)$ absorption medium which giving its capability to detect the incident wavelengths at optimum spectrum for fiber optic which is ranging between $1.3 \mu \mathrm{m}$ and $1.6 \mu \mathrm{m}$ wavelength. APDs are well-known for their high gain due to avalanche multiplication process. Furthermore, the gainbandwidth product in SAGCM APD would introduced an intrinsic layer of multiplication, and a narrow high-doped charge layer [10]. Since, 90s, the studies of APDs [12-14] have been focused on the thin multiplication layer due to its positive effect on the performance of APD. However, there still lack of experimental data on the effect of multiplication layer width on APD performance. On the other hand, to avoid time-consuming and cost inefficient of fabrication and wafer grow [17] of InGaAs/InP APD, a precise simulation can provide equally accurate data (as experimental data) for the purpose of performance enhancement and design optimization of APDs. Thus, an extensive attempt and investigation have been done in this paper to simulate and model the SAGCM InGaAs/InP APD. The electrical characteristic such as voltage breakdown, as well as photo generation and gain, will be analyzed by varying the multiplication layer width (MLW) at three different $(1.8 \mu \mathrm{m}, 2.0 \mu \mathrm{m}, 2.2 \mu \mathrm{m})$ absorption layer widths (ALW). The study of impact ionization and multiplication gain [7] confirmed that the impact ionization of charged carriers dominates the avalanche multiplication by which one charged carrier losses its energy by creating other charged carriers. In addition, by taking advantage of high electric field region of APD, the impact ionization process can generate more charged carriers. When the impact ionization process is self-sustained, photocurrent reaches to infinity where avalanche breakdown occurs. Theoretically, M. H. Wood and W. C. Johnson [10] described two methods for APD gain calculation which are steady-state point of view and transit case where initiation is done by the fixed number of carriers. In this paper, the steady-state point of view method has been adapted for analyzing APD gain. In this method, the current distribution of electrons and holes are examined respectively, by taking into account the high electric field region and collection efficiency of APD. In this work, a list of semiconductor material parameters which is suitable for (SAGCM) InGaAs/InP APD design using Silvaco ATLAS framework was fully established by correlating the simulation results to the experimental results. Further work was carried out by simulating the APDs with different ALW and MLW.

\section{Device Structure and Simulation Model}

In order to construct and simulate the SAGCM InGaAs/InP APD model, 2D Silvaco Technology Computer Aided Design (Silvaco- 
TCAD) was utilized. The ATLAS framework integrated BLAZE and Luminous was used to simulate and analyse the heterojunction interface and electric field, as well as the photocurrent and extracting gain data. The device cross-section of the main design of simulated APD with ALW $=2.0 \mu \mathrm{m}$ and $\mathrm{MLW}=0.6 \mu \mathrm{m}$ is shown in the Figure 1. As the figure represents, an APD includes several layers and electrodes with two anodes on top and a cathode at the bottom of the structure. These contact points were used to apply the reverse bias to the APD where the anode was connected to the negative port and cathode to the positive port of the power supply).

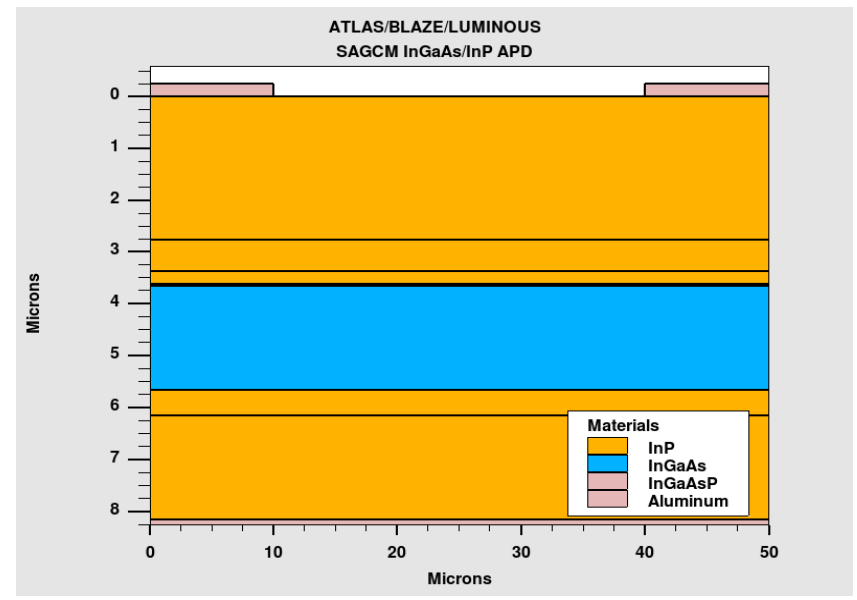

Fig 1: Cross section view of main design of simulation APD

The fabrication of SAGCM InGaAs/InP APD, as shown in the figure 1 , start with the high doped n-type InP substrate layer and mild doped $\left(\sim 7 \times 10^{16}\right)$ InP buffer layer on top of it. The next step is to grow undoped $\left(1 \times 10^{15}\right) \mathrm{InGaAs}$ absorption layer followed by InP charged layer. The purpose of the charge layer is to regulate the electric field distribution in absorption and multiplication layers. To avoid charge accumulation in heterojunction interface, several layers of InGaAsP quaternary compounds sandwiched between (InGaAs) absorption and (InP) charged layers. Finally, undoped n-type InP multiplication layer and high doped p-type InP layer are fabricated sequentially. Table 1 presents the structure parameters of (SAGCM) InGaAs/InP APD in the simulation. The device structure is similar to reference [5] except for the thicknesses of the multiplication layer and absorption layer, which were optimized to $0.6 \mu \mathrm{m}$ and $2.0 \mu \mathrm{m}$ respectively.

Table 1: Structure parameters of SAGCM InGaAs/InP APD

\begin{tabular}{|c|c|c|}
\hline Layers & Thickness (um) & Doping $\left(\mathrm{cm}^{-3}\right)$ \\
\hline $\mathrm{P}^{+}$-InP & 2.77 & $4 \times 10^{17}$ \\
\hline i-InP Multiplication & 0.6 & $1 \times 10^{15}$ \\
\hline $\mathrm{n}^{+}$-InP Charged & 0.25 & $1 \times 10^{17}$ \\
\hline InGaAsP Grading & $0.012 \times 3$ & $1 \times 10^{16}$ \\
\hline i-In ${ }_{0.53} \mathrm{Ga}_{0.47}$ As Absorption & 2.0 & $1 \times 10^{15}$ \\
\hline InP Buffer & 0.5 & $6.6 \times 10^{16}$ \\
\hline InP Substrate & 2.0 & $3 \times 10^{18}$ \\
\hline
\end{tabular}

Generally, in any charge area with enough reverse bias applied, the electric field will be sufficiently high to speed up the free carriers up to the point at which they will be able to acquire enough energy to generate additional free carrier pairs by collision with the atoms of the material [21]. In order to gain enough energy, two important conditions have to occur: High electric field which results in a high concentration difference between highly pdoped InP diffusion layer and undoped n-type InP multiplication layer; The distance between two collisions has to be short enough to allow carriers (holes) gain sufficient ionization energy. Therefore, the generation rate of free carrier pairs will be high enough to initiate the avalanche process and eventually breakdown, which in this point reverse bias voltage define as breakdown voltage $\left(V_{b}\right)$. As such, for simulation of the avalanche process, it is necessary to calculate the generation rate of electrons and holes $(G)$ due to impact ionization as a function of electric field. In Silvaco ATLAS this calculation is performed by [34]

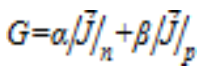

where $J_{n, p}$ is the electrons and holes current density. The impact ionization is modeled with the Selberherr model using the following equations:

$\alpha=\alpha_{n} \exp \left[\left(-\frac{E_{\mathrm{mar}}}{E}\right)^{C_{\mathrm{m}}}\right]$

$\beta=\alpha_{\mathrm{p}} \exp \left[\left(-\frac{E_{\mathrm{w} \mu r}}{E}\right)^{C_{\mathrm{pt}}}\right]$

where the $\mathrm{E}$ is the electric field, $\alpha_{n}$ and $\alpha_{p}$ are impact ionization coefficients for electron and hole, $E_{n c f}$ and $E_{p c f}$ are a critical field of electron and hole, and $C_{n}, C_{p}$ are equation`s exponents which in this work assumed as unity. In addition, parameters that contribute to the dark current and photocurrent mechanisms, such as generation-recombination, band to band tunneling current and carriers lifetime were considered to simulate the APD photocurrent and dark current. The summary of the optimized material parameters based on trial and error techniques for $\mathrm{InP}$ and InGaAs materials were listed in Table 2. The basic material parameters, such as electron and hole mobility, saturation velocity, and electron affinity are integrated with BLAZE/ATLAS and kept as defaults. In order to simulate the performance of the modeled APD under an illuminated condition, an ATLAS Gaussian form of light beam was used and introduced at the top center of APD structure with the maximum intensity of $0.4 \mathrm{~cd}$. This light beam would provide a similar illumination condition as the real optical fiber spectrum [31].

\section{Results and Discussion}

Figure 2 shows the comparison of the current-voltage $(I-V)$ plot between an experimental work done in the reference [5] and the modeled SAGCM InGaAs/InP APD with the similar structures, under a reversed bias voltage for both dark and illuminated condition. As it can be seen, the level of the experimental dark current slightly higher than the simulated dark

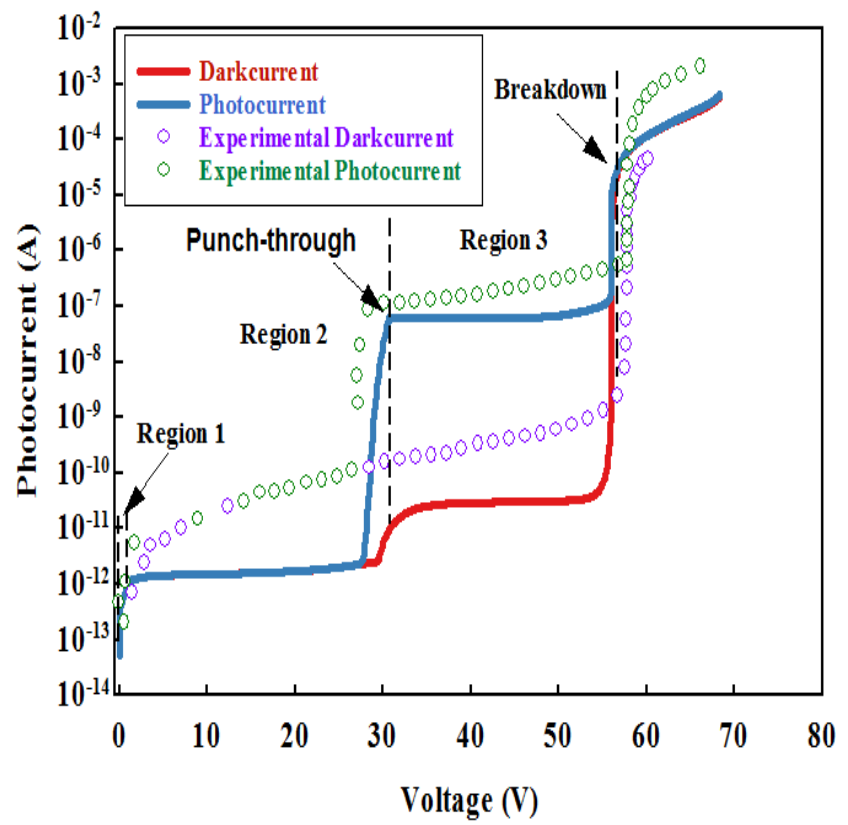

Fig 2: Simulated photocurrent and dark current for main APD design compare with experimental dark/photocurrent. 
Table 2: Numerical parameters at $300 \mathrm{~K}$

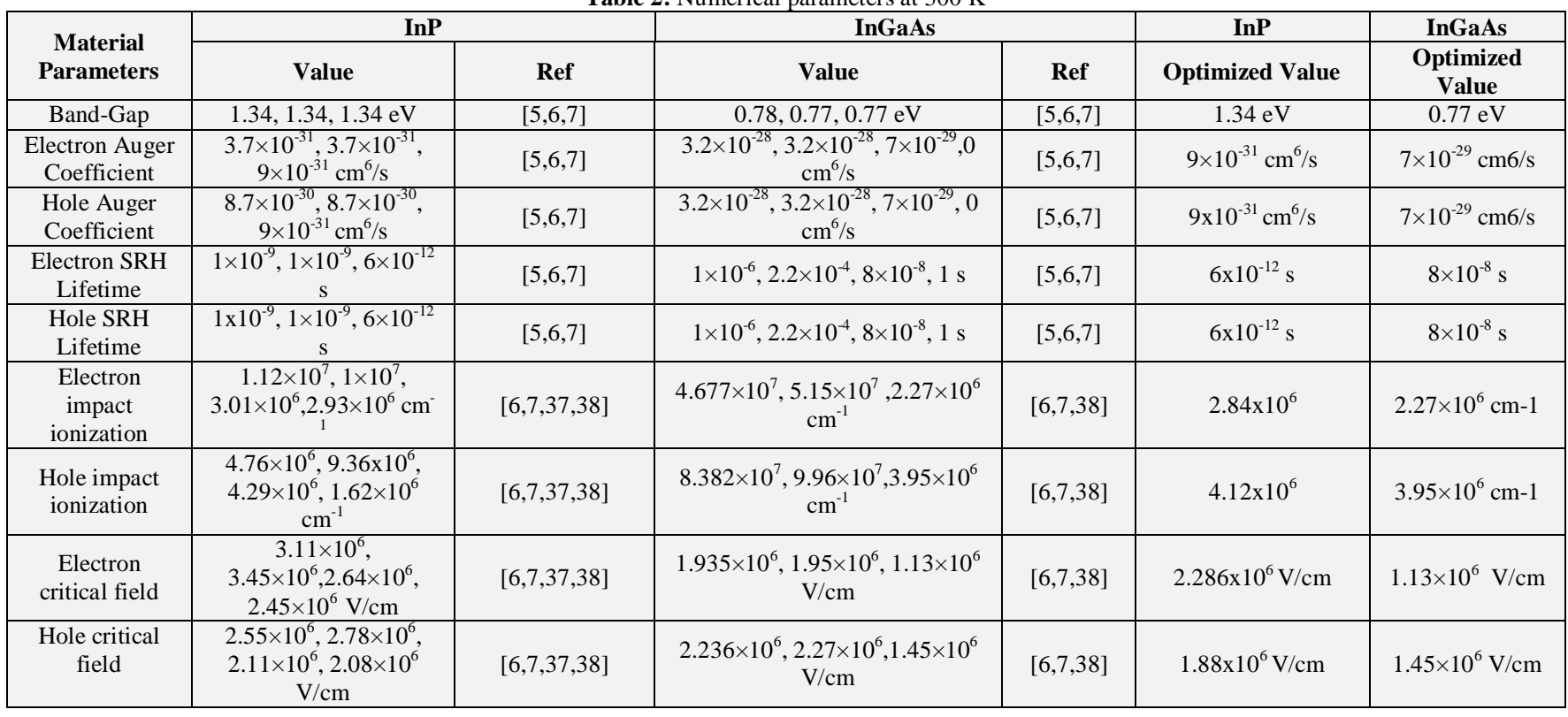

current due to surface leakage current [39][40], while the other characteristics such as multiplication and breakdown are same. It can also be observed that the variation of dark current and photocurrent in APD from zero to breakdown voltage $\left(V_{b}\right)$ can be divided into three primary operation regions. In region 1 , neither the charge layer or abruption layer have been depleted, the collection efficiency of the photocurrent case is virtually zero and dark current is due to thermally excited carriers in InP. In region 2, charge layer has been fully depleted, but the generated carriers still not able to transmit to the high electric field zone, therefore, there is no avalanche process, and thus ADP operates as a PIN photodiode. In this mode, the dark current is due to generated carrier in narrow bandgap InGaAs absorption layer, whereas, the collection efficiency of the illuminated case is near unity due to the low absorption effect.

Furthermore, the depletion zone expands to the absorption layer, which in this point; the reverse bias voltage defines as the punchthrough voltage $\left(V_{p}\right)$. For region 3, the actual operation of APD begins. In this region, the $V_{p}$ is high enough to deplete the (InGaAs) absorption layer entirely, generated carriers reaching to high electric field multiplication layer. Thus, the impact ionization process will initiate, device act as an APD and avalanche multiplication goes to infinity where the breakdown occurs. The $V_{p}$ and the breakdown voltage, (as a voltage that the avalanche gain is infinity) is influenced by structure parameters such as doping profile and layers thickness.

The photocurrent-voltage $\left(I_{p h^{-}}-V\right)$ curves for all the simulated structures at the temperature of $300 \mathrm{~K}$ are shown in Figure 3. The figure indicates that $V_{p}$ and $V_{b}$ is corresponding to the MLW. Moreover, for the thin MLW the distance between $V_{b}$ and $V_{p}$ is more significant than the APD with large MLW, and this is because, the absorption layer of APD with thin MLW requires lower $V_{p}$ to be fully depleted. Therefore, it can be seen from the Figure 3 that the APD with thin MLW has more extended operation region.

In order to achieve multiplication gain $(M)$, the ratio of photocurrent $\left(I_{p h}\right)$ over primary current $\left(I_{p r}\right)$ was used (in logarithmic scale) as shown in the following equation

$M=\frac{I_{\text {BH }}}{I_{\mathrm{IFF}}}$

The $I_{p h}$ for each point is directly achieved from $I_{p h}-V$ curve that been simulated and shown in Figure 3. On the other hand, to have the primary current in the same point (as achieved for $I_{p h}$ ) the liner form of trendline equation from $I_{p h^{-}} V$ curve must be considered (to find the pattern of $I_{p h}-V$ curve) which is in the following form

$y=m x+c$

where $\mathrm{m}$ and $\mathrm{c}$ are the slope and intercept of the line respectively. Now by replacing $y$ with $I_{p r}$ and $x$ with each voltage points $(V)$ in $I_{p h}-V$ curve for all three operating regions of APD, the $I_{p r}$ of APD will be defined as

$I_{p Y}=m V+c$

In this way, not only primary current $\left(I_{p r}\right)$ based on all voltage points were taken into account, it can also guaranty that the collection efficiency of the APD has been considered and effect the multiplication gain curve. The curve of multiplication gain versus reverse bias voltage $(M-V)$ with vary MLW (for three different

ALW) while the doping profile and other numerical parameters kept unchanged are presented in the Figure 4. As discussed earlier the actual operation of APD is in the voltage point at which the InGaAs absorption layer just start to be depleted (which known as punch-through voltage). Therefore, the $M-V$ curves shown in Figure 4, are started from unity at which corresponds to the punchthrough voltage and below unity point has been ignored since the APD does not have any operation regarding multiplication gain.

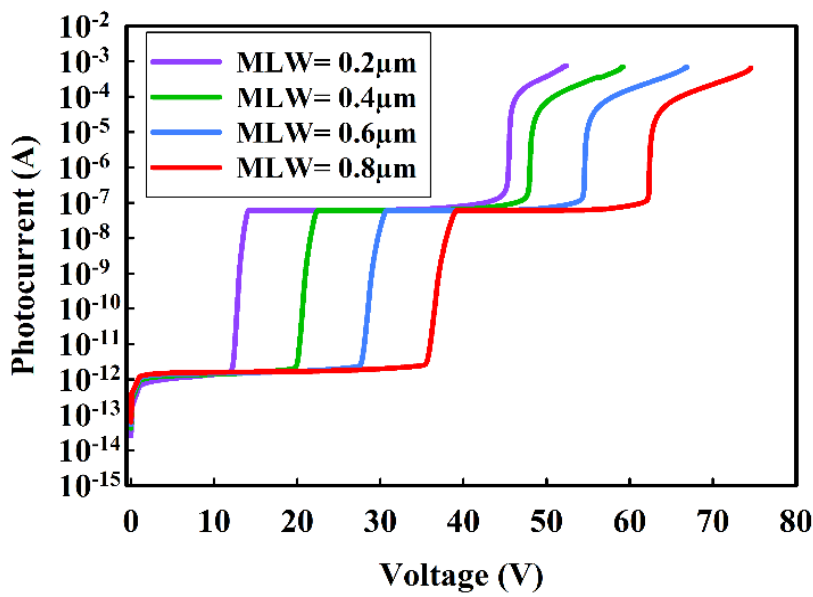

(a) 


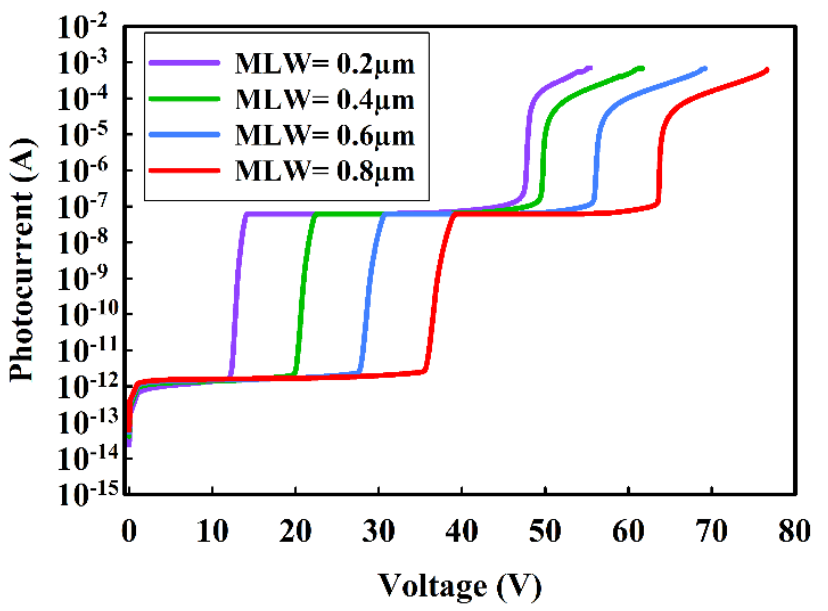

(b)

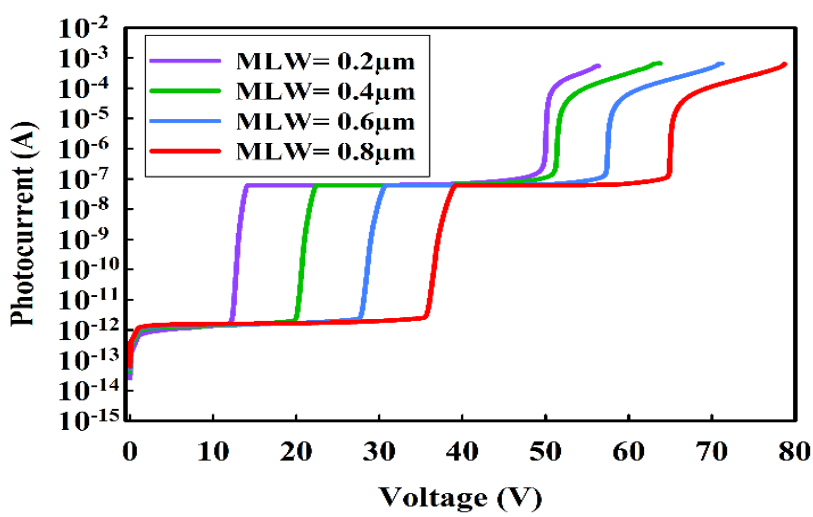

(c)

Fig 3: Photocurrent $(I)$ versus voltage $(V)$. (a) Shows the $I$ - $V$ curve of $\mathrm{ALW}=1.8 \mu \mathrm{m}$ (b) is the $I-V$ curve of $\mathrm{ALW}=2.0 \mu \mathrm{m}$ and (c) is the $I-V$ curve of $\mathrm{ALW}=2.2 \mu \mathrm{m}$. . In all three graphs the MLW vary from $0.2 \mu \mathrm{m}$ to $0.8 \mu \mathrm{m}$.

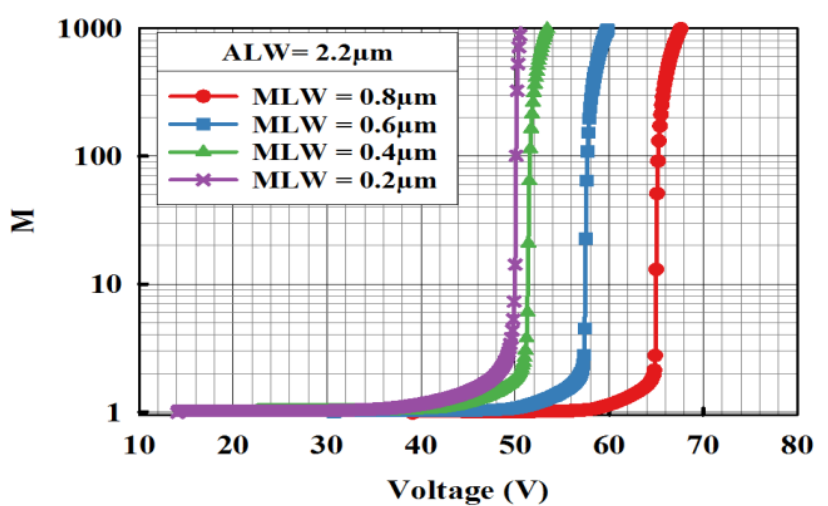

(a)

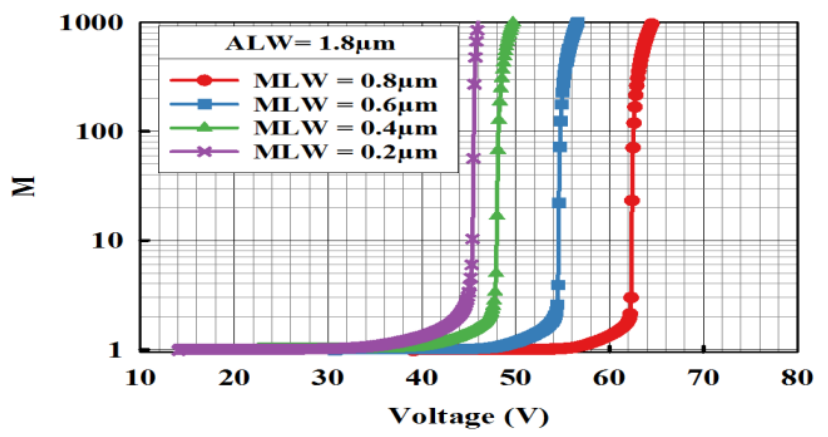

(b)

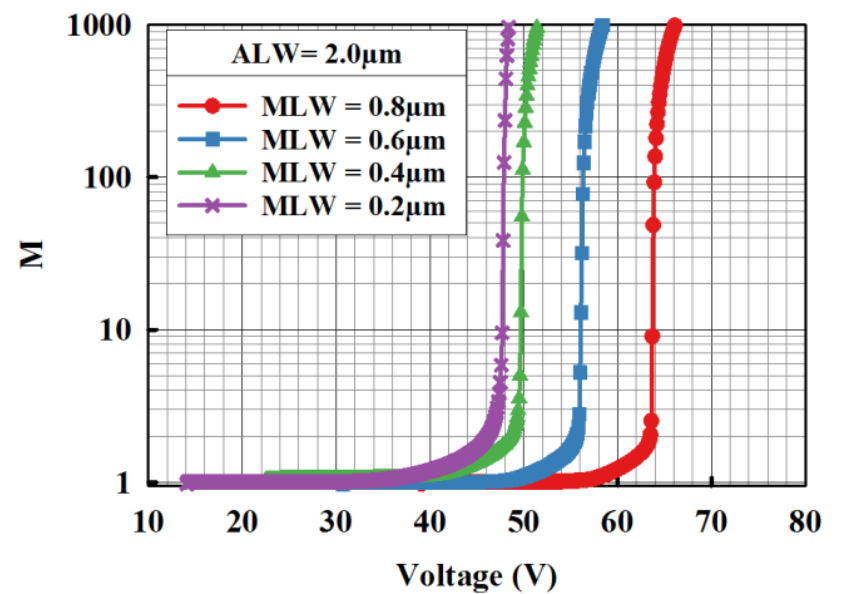

(c)

Fig 4: Multiplication gain $(M)$ versus Voltage $(V)$. (a) Shows the $M-V$ curve of ALW=1.8 $\mu \mathrm{m}$, (b) is the $M-V$ curve of $\mathrm{ALW}=2.0 \mu \mathrm{m}$ and (c) is the $M-V$ curve of $\mathrm{ALW}=2.2 \mu \mathrm{m}$. In all three graphs the MLW vary from $0.2 \mu \mathrm{m}$ to $0.8 \mu \mathrm{m}$.

\section{Conclusion}

Modeling of the multiplication gain for SAGCM InGaAs/InP avalanche photodiodes designed structure has been carried out through the simulation of three ALW with varying MLW. The material parameters needed for the simulation of the APD were fully established through a comprehensive correlation between the simulation and experimental results. From the simulation results, the APD characteristics, such as the multiplication gain and the voltage breakdown, were correlated to the characteristic variations of the APD. It was also found that the thickness of the InP multiplication layer is critical for characterization of avalanche breakdown and punch-through voltage. Furthermore, it affects the APD operation region since it can dominate the distance between punch-through voltage and breakdown voltage.

\section{Acknowledgement}

The authors gratefully acknowledge the Tenaga Nasional Berhad (TNB) Seeding fund (Project code: U-TG-RD-18-04) for the access to the simulation software and the UNITEN BOLD grant (Project code: 10289176/SMART/2018/02).

\section{References}

[1] Bhattacharya, P. (2009). Semiconductor optoelectronic devices. New Delhi: Prentice Hall India.

[2] Fukuda, M. (1999). Optical semiconductor devices. New York [u.a.]: Wiley.

[3] Saleh, B. and Teich, M. (2007). Fundamentals of photonics. Hoboken,NJ: Wiley.

[4] Senior, J. and Jamro, M. (2009). Optical fiber communications. Harlow, England: Financial Times/Prentice Hall.

[5] Xu, J., Chen, X., Wang, W. and Lu, W. (2016). Extracting dark current components and characteristics parameters for InGaAs/InP avalanche photodiodes. Infrared Physics \& Technology, 76, pp.468-473.

[6] Parks, J., Smith, A., Brennan, K. and Tarof, L. (1996). Theoretical study of device sensitivity and gain saturation of separate absorption, grading, charge, and multiplication InP/InGaAs avalanche photodiodes. IEEE Transactions on Electron Devices, 43(12), pp.2113-2121.

[7] Zeng, Q., Wang, W., Wen, J., Xu, P., Hu, W., Li, Q., Li, N. and Lu, W. (2014). Dependence of dark current on carrier lifetime for InGaAs/InP avalanche photodiodes. Optical and Quantum Electronics, 47(7), pp.1671-1677

[8] Susa, N., Nakagome, H., Ando, H. and Kanbe, H. (1981). 
Characteristics in InGaAs/InP avalanche photodiodes with separated absorption and multiplication regions. IEEE Journal of Quantum Electronics, 17(2), pp.243-250.

[9] Zhao, Y., Zhang, D., Qin, L., Tang, Q., Wu, R., Liu, J., Zhang, Y., Zhang, H., Yuan, X. and Liu, W. (2011). InGaAs-InP avalanche photodiodes with dark current limited by generation-recombination. Optics Express, 19(9), p.8546.

[10] M. Woods, W. Johnson, and M. Lampert, "Use of a Schottky barrier to measure impact ionization coefficients in semiconductors,"Solid-State Electronics, vol. 16, no. 3, pp. 381394, 1973

[11] Ma, J., Bai, B., Wang, L., Tong, C., Jin, G., Zhang, J. and Pan, J. (2016). Design considerations of high-performance InGaAs/InP single-photon avalanche diodes for quantum key distribution. Applied Optics, 55(27), p.7497.

[12] Acerbi, F., Tosi, A. and Zappa, F. (2013). Growths and diffusions for InGaAs/InP single-photon avalanche diodes. Sensors and Actuators A: Physical, 201, pp.207-213.

[13] C. Ma, Characterization and modelling of SAGCM InP/InGaAs avalanche photodiodes for multigigabit optical fiber communications. Ottawa: National Library of Canada, 1996.

[14] H. T. J. Meier, Design, characterization and simulation of avalanche photodiodes. Zürich: ETH, 2011.

[15] Saleh, M., Hayat, M., Sotirelis, P., Holmes, A., Campbell, J., Saleh, B. and Teich, M. (2001). Impact-ionization and noise characteristics of thin III-V avalanche photodiodes. IEEE Transactions on Electron Devices, 48(12), pp.2722-2731.

[16] Welch, D., Kish, F., Melle, S., Nagarajan, R., Kato, M., Joyner, C., Pleumeekers, J., Schneider, R., Back, J., M. and Mehuys, D. (2007). Large-Scale InP Photonic Integrated Circuits: Enabling Efficient Scaling of Optical Transport Networks. IEEE Journal of Selected Topics in Quantum Electronics, 13(1), pp.22-31.

[17] P. S. A. Roslan, P. J. Ker, I. Ahmad, J. Pasupuleti, and P. Z. Fam "Modeling and simulation of InAs photodiode on electric field profile and dark current characteristics," 2016 IEEE International Conference on Semiconductor Electronics (ICSE), 2016.

[18] O'Reilly, J. and Fyath, R. (1988). Analysis of the influence of dark current on the performance of optical receivers employing superlattice APDs. IEE Proceedings J Optoelectronics, 135(2), p. 109.

[19] Fyath, R. and O'Reilly, J. (1989). Performance degradation of APDoptical receivers due to dark current generated within the multiplication region. Journal of Lightwave Technology, 7(1), pp.62-67.

[20] Ma, C., Deen, M. and Tarof, L. (1995). Multiplication in separate absorption, grading, charge, and multiplication InP-InGaAs avalanche photodiodes. IEEE Journal of Quantum Electronics, 31(11), pp.2078-2089.

[21] An, S. and Deen, M. (2000). Low-frequency noise in single growth planar separate absorption, grading, charge, and multiplication avalanche photodiodes. IEEE Transactions on Electron Devices, 47(3), pp.537-543.

[22] Chen, Y., Wun, J., Wu, S., Chao, R., Huang, J., Jan, Y., Chen, H., Ni, C., Chang, H., Chou, E. and Shi, J. (2018). Top-Illuminated In0.52A10.48As-Based Avalanche Photodiode With Dual Charge Layers for High-Speed and Low Dark Current Performances. IEEE Journal of Selected Topics in Quantum Electronics, 24(2), pp.1-8.

[23] Tien-Pei Lee, Burrus, C. and Dentai, A. (1981). InGaAs/InP p-i-n photodiodes for lightwave communications at the 0.95-1.65 $\mu \mathrm{m}$ wavelength. IEEE Journal of Quantum Electronics, 17(2), pp.232238.

[24] P. Yuan, K. Anselm, C. Hu, H. Nie, C. Lenox, A. Holmes, B. Streetman, J. Campbell, and R. Mcintyre, "A new look at impact ionization-Part II: Gain and noise in short avalanche photodiodes,"IEEE Transactions on Electron Devices, vol. 46, no. 8, pp. 1632-1639, 1999.

[25] P. Yuan, C. Hansing, K. Anselm, C. Lenox, H. Nie, A. Holmes, B. Streetman, and J. Campbell, "Impact ionization characteristics of III-V semiconductors for a wide range of multiplication region thicknesses," IEEE Journal of Quantum Electronics, vol. 36, no. 2, pp. 198-204, 2000.

[26] G. M. Williams, M. Compton, D. A. Ramirez, M. M. Hayat, and A. S. Huntington, "Multi-Gain-Stage InGaAs Avalanche Photodiode With Enhanced Gain and Reduced Excess Noise," IEEE Journal of the Electron Devices Society, vol. 1, no. 2, pp. 54-65, 2013

[27] R. Fyath and J. Oreilly, "Performance degradation of APD-optical receivers due to dark current generated within the multiplication region,” Journal of Lightwave Technology, vol. 7, no. 1, pp. 62-67,
1989.

[28] J. Chen, Z. Zhang, M. Zhu, J. Xu, and X. Li, "Optimization of InGaAs/InAlAs Avalanche Photodiodes," Nanoscale Research Letters, vol. 12, no. 1, 2017.

[29] J. Tu, Y. Zhao, K. Wen, Q. Li, and Y. Li, "The Determination of Unity Gain for InGaAs/InP Avalanche Photodiodes With Excess Noise Measurements," IEEE Photonics Technology Letters, vol. 29, no. 8, pp. 671-674, 2017.

[30] M. H. Mun, S. W. Jung, H. Kang, D. Kim, H. J. Kim, S. H. Lee, and H. Park, "Design and Simulation Result of N Substrate Reverse Type Avalanche Photodiode (APD)," IEEE Transactions on Nuclear Science, vol. 56, no. 3, pp. 1046-1050, 2009.

[31] J. K. Park and I. Yun, "Modeling of avalanche gain for high-speed InP/InGaAs avalanche photodiodes," 2008 IEEE International Conference on Electron Devices and Solid-State Circuits, 2008.

[32] G.-F. D. Betta, L. Pancheri, M. Boscardin, G. Paternoster, C. Piemonte, N. Cartiglia, F. Cenna, and M. Bruzzi, "Design and TCAD simulation of double-sided pixelated low gain avalanche detectors," Nuclear Instruments and Methods in Physics Research Section A: Accelerators, Spectrometers, Detectors and Associated Equipment, vol. 796, pp. 154-157, 2015.

[33] Silvaco Standard Simulation, Two-Dimensional Device Simulation of InGaAs/InP Avalanche Photodiode, 2014

[34] V. Palankovski, Analysis and simulation of heterostructure devices. Place of publication not identified: Springer Verlag Gmbh, 2012.

[35] ATLAS User's Manual-Device Simulation Software Silvaco, 2016

[36] Marshall, A., David, J. and Tan, C. Impact Ionization in InAs Electron Avalanche Photodiodes. IEEE Transactions on Electron Devices, 57(10), pp.2631-2638. 2010

[37] Saleh, M., Hayat, M., Sotirelis, P., Holmes, A., Campbell, J., Saleh, B., \& Teich, M. (2001). Impact-ionization and noise characteristics of thin III-V avalanche photodiodes. IEEE Transactions on Electron Devices,48(12), 2722-2731. doi:10.1109/16.974696

[38] S. L. Chuang, Physics of photonic devices. Hoboken, NJ: John Wiley \& Sons, 2009

[39] Jackson, J. C., Hurley, P. K., Lane, B., Mathewson, A., \& Morrison, A. P. (2002). Comparing leakage currents and dark count rates in Geiger-mode avalanche photodiodes. Applied Physics Letters,80(22), 4100-4102. doi:10.1063/1.1483119

[40] R. Yeats and S. H. Chiao, "Leakage current in InGaAsP avalanche photodiodes," Applied Physics Letters, vol. 36, no. 2, pp. 167-170, 1980 . 